Discussion Comprehensive guidance beyond patient handling policies and training is needed for prevention of work-related ULDs that address physical and psychosocial exposures. Work organisation changes such as increased control over work, scheduling and rest breaks emerge as simple interventions to manage physical and psychosocial exposures. Examples will be provided.

\section{OCCUPATIONAL HEALTH HAZARDS, HEALTH PROBLEMS ENCOUNTERED AND PERSONAL PROTECTIVE EQUIPMENT USED IN HEALTHCARE WORKERS IN HOSPITALS, THAILAND}

Kongtip* 1Noppanun - Nankongnab-2Pimpan Silpasuwan, ${ }^{3}$ Mathuros Tipayamongkholgul, ${ }^{2}$ Orawan Kaewboonchoo, ${ }^{4}$ Pipat Luksamijarulkul, ${ }^{5}$ Susan Woskie. 'Department of Occupational Health and Safety, Faculty of Public Health, Mahidol University, Bangkok, Thailand; '2Department of Public Health Nursing, Faculty of Public Health, Mahidol University, Bangkok, Thailand; ${ }^{3}$ Department of Epidemiology, Faculty of Public Health, Mahidol University, Bangkok, Thailand; ' Department of Microbiology, Faculty of Public Health, Mahidol University, Bangkok, Thailand; ${ }^{5}$ College of Health Sciences, University of Massachusetts Lowell, Lowell, MA, USA

\subsection{6/oemed-2018-ICOHabstracts.990}

Introduction Healthcare workers usually expose to chemical, physical, biological and ergonomic hazards in their everyday life. The objectives of this cross-sectional study are to evaluate chemical, physical, biological and ergonomic hazards and health problems of healthcare workers in hospitals, accidents, contact with chemicals and body fluids and personal protective equipment used among healthcare workers in Thailand.

Methods The self-administered and interviewed questionnaires were distributed or collected from healthcare workers proportion to size of five hospital staffs in five regions of Thailand.

Results Healthcare workers have been working very hard for 9 to 11 hours/day on average, including overtime work ranging 13-18 hours/day. More than half of the inpatient, surgery and anaesthesia, nutrition service department did shift work. Healthcare workers at nutrition service department reported highest musculoskeletal disorders, respiratory symptoms and hearing loss than other departments. In surgery and anaesthesia department, they reported highest skin problem due to highest chemical exposure and biological hazards; they exposed to radiation, vibration from equipment and tools, glare and inadequate lighting leading to eye irritation, eye pain and blur vision and reported highest non- specific symptoms. The regular compliance with safety rule and protocol of healthcare workers were not so high, inpatient $(65.8 \%)$, outpatient (65.9\%), surgery and anaesthesia (77.6\%), nutrition service $(78.4 \%)$ and hospital supporting service (66.9\%). The regular correct working posture of them was not high either (51\% or lower). Regarding hazard control and personal protective equipment provided in workplace, inpatient department reported highest, followed by surgery and anaesthesia, outpatient, nutrition service and hospital supporting service.

Conclusion The healthcare workers were exposed to many occupational health hazards, high risk of accidents, working very hard; they need more attention to reduce or control the occupational health hazards in the workplace. 74 CARDIOVASCULAR RISK FACTORS AND COMORBIDITIES
IN HEALTH CARE WORKERS - IS THERE A GENDER DIFFERENCE?

Andrea Kaifie, Thomas Kraus, Andre Esser. Institute of Occupational and Social Medicine, Medical Faculty, RWTH Aachen University, Germany

\subsection{6/oemed-2018-ICOHabstracts.991}

Introduction Health care workers experience the implications of health and disease every day, including the crucial role of health-damaging behaviour on morbitity and mortality. This study was conducted to analyse the health behaviour and comorbidities in health care workers with focus on gender differences.

Methods In this study we analysed the clinical data of $n=273$ health care workers (166 males, 107 females) who presented in our occupational medicine outpatient unit. The focus of this study was to assess cardiovascular risk factors, such as obesity, smoking, or physical inactivity as well as diseases of the musculosceletal system and mental illness.

Result Female health care workers presented a higher trend of smoking in comparison to male health care workers $(43,0 \%$ vs $32,5 \%)$. Furthermore, female health care workers showed significant less physical activity in comparison to their male colleagues $(49,0 \%$ vs $71,8 \%, p>0,001)$. Musculosceletal diseases were common in both groups $(19,6 \%$ for female vs $18,1 \%$ for male), but mental illness was significantly more frequent in females $(6,5 \%$ vs $0,0 \%$ for males, $\mathrm{p}<0,05)$.

Discussion Female health care workers need special consideration in the implementation of preventive measures to reduce health-damaging behaviour. The higher proportion of mental illness in female in comparison to male workers might be due to a selection bias.

\section{SEASONAL INFLUENZA VACCINATION IN HEALTH CARE WORKERS AND STUDENTS. SURVEY IN A LARGE ITALIAN UNIVERSITY HOSPITAL}

${ }^{1} \mathrm{M}$ Belingheri ${ }^{*},{ }^{2,3} \mathrm{MA}$ Riva, ${ }^{1} \mathrm{M}$ Turato, ${ }^{3} \mathrm{G}$ De Vito, ${ }^{3} \mathrm{MI}$ D'Orso, ${ }^{2} \mathrm{R}$ Latocca, ${ }^{3} \mathrm{G}$ Cesana. ${ }^{1}$ School of Specialisation in Occupational Health, University of Milan, Milan, Italy; ${ }^{2}$ Occupational Health Unit, San Gerardo Hospital, Monza, Italy; ${ }^{3}$ School of Medicine and Surgery, University of Milan Bicocca, Milan, Italy

\subsection{6/oemed-2018-ICOHabstracts.992}

Introduction Vaccination is an important measure for preventing influenza. Its importance in healthcare settings is twofold: it does not only protects Health Care Workers (HCWs) - possibly reducing disease-related work absenteeism and the consequent disruption of health services - but vulnerable patients too. The aim of the study was to evaluate the influenza vaccination coverage among HCWs and students in a large Italian university hospital.

Methods We collected data on influenza vaccination among HCWs and healthcare students in the period 2012-2016. Data included sex, age, work unit, and job title (HCWs)/ degree course (students). We applied chi-square test and t-test for statistical comparisons. The level of significance adopted was $5 \%$. 